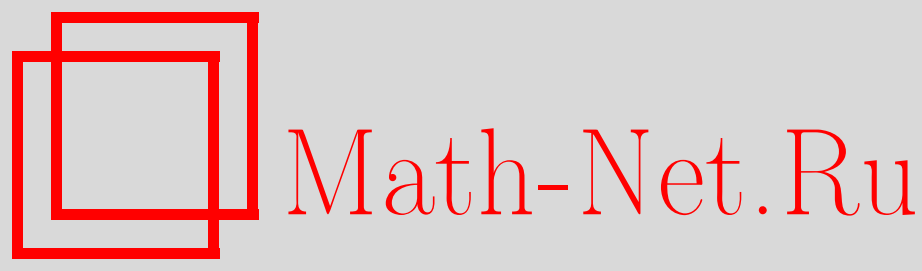

Д. В. Быков, А. А. Славнов, Матричные и векторные модели в пределе сильной связи, ТМФ, 2008, том 155, номер 2, 236-243

DOI: https://doi.org/10.4213/tmf6207

Использование Общероссийского математического портала Math-Net.Ru подразумевает, что вы прочитали и согласны с пользовательским соглашением http://www . mathnet.ru/rus/agreement

Параметры загрузки:

IP : 54.198 .67 .100

26 апреля 2023 г., 15:58:42

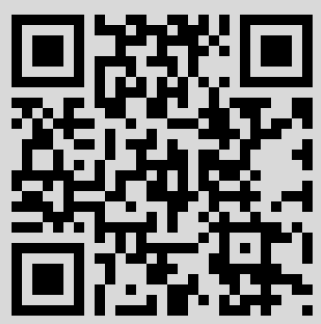




\title{
ФИЗИКА
}

Том 155, № 2

май, 2008

2008 г.

\author{
Д. В. Быков*, А. А. Славнов ${ }^{\dagger}$
}

\section{МАТРИЧНЫЕ И ВЕКТОРНЫЕ МОДЕЛИ В ПРЕДЕЛЕ СИЛЬНОЙ СВЯЗИ}

\begin{abstract}
Рассматриваются матричные и векторные модели в пределе больших $N$ : изучаются $(N \times N)$-матрицы и векторы с $N^{2}$ компонентами. Для случая нульмерной модели $(D=0)$ доказано, что в пределе сильной связи $(g \rightarrow \infty)$ статистические суммы обеих моделей совпадают с точностью до коэффициента. Это справедливо также при $D=1$.
\end{abstract}

Ключевые слова: матричные модели, векторные модели, $1 / N$-разложение.

\section{1. ПОСТАНОВКА ЗАДАЧИ}

Матричные модели находят применение в разных областях физики, однако для нас основной мотивировкой является возможность их использования в квантовой теории поля. В частности, как было показано 'т Хофтом [1], при соответствующей нормировке констант связи разложение функций Грина (и статсуммы) по $1 / N$ отвечает в терминах ленточных диаграмм Фейнмана разложению по родам римановой поверхности, на которой данный граф может быть нарисован без самопересечений. Однако если даже зафиксировать род поверхности, отвечающих ему ленточных графов остается бесконечное множество и в большинстве интересных случаев, к сожалению, пока нет алгоритма их суммирования.

Предел $N \rightarrow \infty$ представляет также интерес с точки зрения исследования $\mathrm{AdS} / \mathrm{CFT-соответствия} \mathrm{[2].} \mathrm{В} \mathrm{этом} \mathrm{случае} \mathrm{необходимо} \mathrm{рассматривать} \mathrm{предел} N \rightarrow \infty$ при больших константах связи $g$. Эта задача далека от решения не только для калибровочных теорий, но и для более простых скалярных матричных моделей. Исключение составляют случаи $D=0$ и $D=1$, в которых алгоритм суммирования планарных диаграмм был найден [3] для вещественных и эрмитовых матричных моделей. Затем (при $D=0$ ) он был обобщен на случай комплексных матричных моделей [4]. Обзор современного состояния теории нульмерных матричных моделей можно найти в [5].

* Московский государственный университет им. М. В. Ломоносова, Москва, Россия. E-mail: dbykov@mi.ras.ru

${ }^{\dagger}$ Математический институт им. В. А. Стеклова РАН, Москва, Россия. E-mail: slavnov@mi.ras.ru 
Векторные и матричные модели при $D=0,1$ демонстрируют замечательное сходство при $g \rightarrow \infty$. Характер зависимости от константы связи в этом пределе одинаков для векторных и матричных моделей. Это наводит на мысль о возможности подобного совпадения и в более высоких размерностях.

Однако в данной работе мы ограничимся рассмотрением комплексной матричной модели при $D=0$, т.е. рассмотрением статсуммы

$$
\mathcal{Z}(g)=\int d \varphi^{\dagger} d \varphi \exp \left[-\frac{1}{2} \operatorname{tr}\left(\varphi^{\dagger} \varphi\right)-\frac{g}{4 N} \operatorname{tr}\left(\left(\varphi^{\dagger} \varphi\right)^{2}\right)\right],
$$

где $\varphi-(N \times N)$-матрица. Обозначение $d \varphi^{\dagger} d \varphi$ следует понимать следующим образом:

$$
d \varphi^{\dagger} d \varphi \equiv D \varphi \equiv \prod_{i, j} d \operatorname{Re} \varphi_{i j} d \operatorname{Im} \varphi_{i j}
$$

Свободной энергией называется величина, определенная соотношением

$$
\mathcal{E}=-\frac{\ln \mathcal{Z}_{\mathrm{BV}}}{N^{2}}
$$

Будет показано, что при $D=0$ в пределе сильной связи $(g \rightarrow \infty)$ ведущая асимптотика свободной энергии данной матричной модели совпадает с ведущей асимптотикой свободной энергии соответствующей векторной модели. При $D=1$ это также верно, однако необходимо более тщательное рассмотрение этого случая. Особый интерес, конечно, представляют случаи $D \geqslant 2$, однако в этих случаях вопрос о справедливости описанной гипотезы является открытым. Сразу оговоримся, что при $D \geqslant 2$ как в матричной, так и в векторной модели появляются ультрафиолетовые расходимости, поэтому имеет смысл говорить лишь о надлежащим образом регуляризованных теориях.

Отметим, что во всех размерностях линейные векторные модели с взаимодействием $N^{2} F\left(\operatorname{tr}\left(\varphi^{\dagger} \varphi\right) / N^{2}\right)$ являются точно решаемыми, т.е. функции Грина синглетных переменных можно найти в явном виде, так что предполагаемая аналогия между матричными и векторными моделями в пределе сильной связи может помочь анализировать и (гораздо более сложные) матричные модели.

\section{2. $D=0$ БИВЕКТОРНАЯ МОДЕЛЬ}

Для сравнения матричной и векторной моделей прежде всего вычислим статистическую сумму векторной модели. Поясним, что мы понимаем под векторной и бивекторной моделями. Распределение $\rho$ случайных величин $x_{a}(a=1,2, \ldots, M)$ в векторной модели, по определению, зависит лишь от квадрата вектора $\mathbf{x}: \rho=\rho\left(x_{a}^{2}\right)$.

Рассмотрим статсумму

$$
\mathcal{Z}_{\mathrm{BV}}=\int D \varphi \exp \left[-\frac{1}{2} \operatorname{tr}\left(\varphi^{\dagger} \varphi\right)+N^{2} F\left(\frac{1}{N^{2}} \operatorname{tr}\left(\varphi^{\dagger} \varphi\right)\right)\right] .
$$

Несмотря на то что формально эта модель является матричной, на самом деле она векторная, так как если ввести вектор $y_{a}$ с $M=2 N^{2}$ компонентами

$$
y_{i}=\operatorname{Re} \varphi_{i, 1}, \quad y_{N^{2}+i}=\operatorname{Im} \varphi_{i, 1}, \quad i \leqslant N,
$$




$$
y_{i}=\operatorname{Re} \varphi_{i-N, 2}, \quad y_{N^{2}+i}=\operatorname{Im} \varphi_{i-N, 2}, \quad N<i \leqslant 2 N, \ldots,
$$

то распределение будет функцией от $y_{a}^{2}$. Такие модели назовем бивекторными. Они характеризуются тем, что их группа симметрии оказывается значительно шире, чем в матричной модели. Например, в данном случае матричная модель имеет группу $U(1) \times S U(N)_{L} \times S U(N)_{R}$ (умножение на фазовый множитель, левое и правое матричное умножение, соответственно ${ }^{1)}$ ), в то время как векторная модель имеет группу $U\left(2 N^{2}\right)$. Эта значительная симметрия и позволяет найти точные решения бивекторных моделей.

Итак, нас интересует свободная энергия в пределе $N \rightarrow \infty$. Внесем под знак интеграла в (2.1) единицу:

$$
\mathcal{Z}_{\mathrm{BV}}=\int D \varphi d \rho \delta\left(\rho-\operatorname{tr}\left(\varphi^{\dagger} \varphi\right)\right) \exp \left[-\frac{1}{2} \operatorname{tr}\left(\varphi^{\dagger} \varphi\right)+N^{2} F\left(\frac{\rho}{N^{2}}\right)\right] .
$$

Запишем теперь преобразование Фурье для дельта-функции

$$
\mathcal{Z}_{\mathrm{BV}}=\frac{1}{2 \pi} \int D \varphi d \rho d \lambda \exp \left[-\frac{1}{2} \operatorname{tr}\left(\varphi^{\dagger} \varphi\right)+N^{2} F\left(\frac{\rho}{N^{2}}\right)+i \lambda\left(\rho-\operatorname{tr}\left(\varphi^{\dagger} \varphi\right)\right)\right] .
$$

Интеграл по $\varphi$ гауссов, причем он вырождается в произведение интегралов, так как компоненты матрицы $\varphi$ не зацеплены: $\operatorname{tr}\left(\varphi^{\dagger} \varphi\right)=\sum_{i, j}\left|\varphi_{i j}\right|^{2}$. В результате интеграл по $\varphi$ можно вычислить, при этом получим:

$$
\mathcal{Z}_{\mathrm{BV}}=\frac{1}{2 \pi} \int d \rho d \lambda\left(\frac{\pi}{1 / 2+i \lambda}\right)^{N^{2}} \exp \left[N^{2} F\left(\frac{\rho}{N^{2}}\right)+i \lambda \rho\right] .
$$

Интеграл по $\lambda$ находится явно, так как мы можем (при условии $\rho>0$, следующем из определения $\left.\rho=\operatorname{tr}\left(\varphi^{\dagger} \varphi\right)\right)$ замкнуть контур в верхней полуплоскости, при этом внутри контура будет находиться полюс $\lambda=i / 2$, имеющий порядок $N^{2}$. Интеграл будет равен $2 \pi i \times($ вычет в этом полюсе). Итак,

$$
\int d \lambda \frac{e^{i \lambda \rho}}{(1+2 i \lambda)^{N^{2}}}=\frac{1}{\left(N^{2}-1\right) !} \frac{2 \pi}{\rho}\left(\frac{\rho}{2}\right)^{N^{2}} e^{-\rho / 2}
$$

В результате получаем

$$
\mathcal{Z}_{\mathrm{BV}}=\frac{\pi^{N^{2}}}{\left(N^{2}-1\right) !} \int d \rho \frac{1}{\rho} \rho^{N^{2}} \exp \left[N^{2} F\left(\frac{\rho}{N^{2}}\right)-\frac{\rho}{2}\right] .
$$

Сделаем замену $\rho \rightarrow N^{2} \rho$, чтобы свести интегрирование к случаю, когда можно применить метод наискорейшего спуска:

$$
\mathcal{Z}_{\mathrm{BV}}=\frac{\left(N^{2} \pi\right)^{N^{2}}}{\left(N^{2}-1\right) !} \int d \rho \frac{1}{\rho} \exp \left[N^{2}\left(\ln \rho+F(\rho)-\frac{\rho}{2}\right)\right] .
$$

Уравнение для стационарной точки $\rho_{0}$ имеет вид

$$
\frac{1}{\rho_{0}}-\frac{1}{2}+F^{\prime}\left(\rho_{0}\right)=0 .
$$

\footnotetext{
1) Умножение на фазовый множитель не сводится к комбинации левого и правого умножений на унимодулярные матрицы $\varphi^{\prime}=U_{1} \varphi U_{2}^{\dagger}$. Действительно, предположим, что это возможно, и положим $\varphi=I$. Тогда $U_{1} U_{2}^{\dagger}=e^{i \alpha} I$, что, очевидно, не имеет места при $\alpha \neq 0$.
} 
Зададим потенциал $F(\rho)=-g \rho^{2} / 4$. Тогда уравнение $(2.8)$ принимает вид

$$
\frac{1}{\rho_{0}}-\frac{1}{2}-\frac{g}{2} \rho_{0}=0
$$

Положительное решение этого уравнения имеет вид

$$
\rho_{0}=\frac{1}{2 g}(\sqrt{1+8 g}-1) .
$$

В результате получим следующее выражение для статсуммы в пределе большого $N$ :

$$
\mathcal{Z}_{\mathrm{BV}}=\frac{\left(N^{2} \pi\right)^{N^{2}}}{\left(N^{2}-1\right) !} \frac{1}{\rho_{0}}\left(\frac{2 \pi}{N^{2} g\left(\frac{1}{2}+\frac{1}{2-\rho_{0}}\right)}\right)^{1 / 2} \exp \left[N^{2}\left(\ln \rho_{0}-\frac{\rho_{0}}{2}-g \frac{\rho_{0}^{2}}{4}\right)\right] .
$$

Применяя формулу Стирлинга $n ! \approx(n / e)^{n} \sqrt{2 \pi n}$, получаем

$$
\mathcal{Z}_{\mathrm{BV}}=(e \cdot \pi)^{N^{2}} \frac{1}{\rho_{0}}\left(\frac{g}{2}+\frac{g}{2-\rho_{0}}\right)^{-1 / 2} \exp \left[N^{2}\left(\ln \rho_{0}-\frac{\rho_{0}}{2}-g \frac{\rho_{0}^{2}}{4}\right)\right] .
$$

Из $(2.10)$ видно, что $\rho_{0} \sim(2 / g)^{1 / 2} \rightarrow 0$ при $g \rightarrow \infty$. Поэтому единственный возрастающий вклад в свободную энергию дает логарифм, при этом

$$
\mathcal{E}_{0} \underset{g \rightarrow \infty}{\rightarrow} \frac{1}{2} \ln g
$$

\section{3. РЕШЕНИЕ $D=0$ МАТРИЧНОЙ МОДЕЛИ С $\operatorname{tr}\left(\left(\varphi^{\dagger} \varphi\right)^{2}\right)$-ВЗАИМОДЕЙСТВИЕМ}

В данном разделе рассмотрим модель, динамической переменной в которой является комплексная $(N \times N)$-матрица $\varphi$. Будет рассматриваться статсумма

$$
\mathcal{Z}=\int d \varphi^{\dagger} d \varphi \exp \left[-\frac{1}{2} \operatorname{tr}\left(\varphi^{\dagger} \varphi\right)-\frac{g}{4 N} \operatorname{tr}\left(\left(\varphi^{\dagger} \varphi\right)^{2}\right)\right] .
$$

Корреляционные функции операторов вида $\operatorname{tr}\left(\left(\varphi^{\dagger} \varphi\right)^{m}\right)$ были получены в [4]. При этом все планарные функции Грина строятся при помощи функции распределения собственных значений $u(\lambda)$, которая определена на сегменте $\lambda \in[0, \sqrt{z}]$ (здесь $\lambda$ нужно интерпретировать как модуль собственного значения, поэтому $\lambda \geqslant 0$ ) и имеет вид

$$
u(\lambda)=\frac{1}{2 \pi}\left(1+\frac{g z}{2}+g \lambda^{2}\right) \sqrt{z-\lambda^{2}}
$$

где $z=2(\sqrt{1+24 g}-1) /(3 g)$. Заметим, что $z \rightarrow 0$ при $g \rightarrow \infty$. При этом площадь под графиком $u(\lambda)$ всегда равна единице: $\int u(\lambda) d \lambda=1$. В результате, очевидно, что

$$
\lim _{g \rightarrow \infty} u(\lambda)=\delta(\lambda)
$$

где предел понимается, конечно, в смысле обобщенных функций. В пределе $g \rightarrow \infty$, как видно, все собственные значения стягиваются к нулю. Тем не менее, напрямую использовать асимптотику (3.3) для получения статсуммы в пределе большого $g$ невозможно, так как в выражении для свободной энергии присутствует слагаемое 
$\int d \lambda u(\lambda) \ln \lambda$, и логарифм дает сингулярность при $\lambda=0$. Мы предложим иной метод решения комплексной матричной модели, свободный от указанного недостатка, и, используя этот метод, докажем справедливость выдвинутого выше утверждения о равенстве свободных энергий при $D=0$. Применим преобразование Хаббарда-Стратоновича для второго слагаемого в показателе экспоненты:

$$
\mathcal{Z}=\int d \varphi^{\dagger} d \varphi d \eta \exp \left[-\frac{1}{2} \operatorname{tr}\left(\varphi^{\dagger} \varphi\right)-\operatorname{tr} \eta^{2}+i \sqrt{\frac{g}{N}} \operatorname{tr}\left(\eta \varphi^{\dagger} \varphi\right)\right],
$$

где $\eta$ - эрмитова матрица. Теперь выполним гауссово интегрирование по $\varphi$ :

$$
\mathcal{Z}=\int d \eta \exp \left[-\operatorname{tr} \eta^{2}-N \operatorname{tr} \ln \left[-\frac{1}{2} I+i \sqrt{\frac{g}{N}} \eta\right]\right] .
$$

После перехода к интегрированию по (вещественным) собственным значениям $\lambda_{k}$ эрмитовой матрицы $\eta$ интеграл можно находить методом наискорейшего спуска. Несмотря на то что интегрирование происходит по вещественному пространству $\mathbb{R}^{N}$, значения $\lambda_{k}$ в стационарной точке в нашем случае комплексные - это обычная ситуация в методе наискорейшего спуска. При варьировании показателя экспоненты по $\lambda_{k}$ получим следующие уравнения:

$$
\sum_{j=1}^{\prime} \frac{2}{\lambda_{k}-\lambda_{j}}=2 \lambda_{k}+\frac{i \sqrt{g N}}{-1 / 2+i \sqrt{g / N} \lambda_{k}}, \quad k=1,2, \ldots, N .
$$

Перейдем к непрерывному случаю $(N \rightarrow \infty)$, заменяя $\lambda_{k} \rightarrow \sqrt{N} \lambda(x) \quad(x \in[0,1]-$ аналог индекса $k$ в непрерывном случае). При этом получим систему

$$
\begin{aligned}
\mathrm{P} \int_{C} \frac{v(\nu) d \nu}{\lambda-\nu} & =\lambda+\frac{\sqrt{g}}{2} \frac{1}{\sqrt{g} \lambda+i / 2}, \\
\int_{C} v(\nu) d \nu & =1
\end{aligned}
$$

где $v(\lambda)=d x / d \lambda$. Из уравнения в дискретной форме (3.6) следует, что если есть решение $\left\{\lambda_{k}\right\}_{k=1,2, \ldots, N}$, то решением также будет $\left\{-\lambda_{k}^{*}\right\}_{k=1,2, \ldots, N}$, поэтому контур $C$ симметричен относительно оси $O y=\operatorname{Im} \lambda$. Умножим первое уравнение на $1-2 i \sqrt{g} \lambda$ и введем новую функцию $\bar{v}(\lambda)=(1-2 i \sqrt{g} \lambda) v(\lambda)$. Согласно расположению контура $\int_{C} \bar{v}(\lambda) d \lambda=D-$ вещественная величина (так как $\operatorname{tr} \lambda=\int_{C} \lambda u(\lambda) d \lambda-$ мнимая). Получим уравнение

$$
\mathrm{P} \int_{C} \frac{\bar{v}(\nu) d \nu}{\lambda-\nu}=i \sqrt{g}+\lambda(1-2 i \sqrt{g} \lambda)
$$

Будем искать "резольвенту"

$$
\Phi(\lambda) \equiv \int_{C} \frac{\bar{v}(\nu) d \nu}{\lambda-\nu}, \quad \lambda \notin C,
$$

в виде

$$
\Phi(\lambda)=i \sqrt{g}+\lambda(1-2 i \sqrt{g} \lambda)+(A \lambda+B) \sqrt{\left(\lambda+b^{*}\right)(\lambda-b)} .
$$

При $\lambda \rightarrow \infty$ задана асимптотика $\Phi(\lambda) \rightarrow D / \lambda$ (это ясно из определения резольвенты и условия нормировки, см. выше), однако так как $D$ нам не известна, мы используем 
следующие условия: коэффициенты при $\lambda^{2}, \lambda^{1}, \lambda^{0}$ обращаются в нуль и

$$
\int_{C} v(\nu) d \nu=\int_{C} \frac{\bar{v}(\nu) d \nu}{1-2 i \sqrt{g} \nu}=1 .
$$

Первые три условия переписываются в виде

$$
\begin{gathered}
-2 i \sqrt{g}+A=0, \\
1+B+i \sqrt{g}\left(b^{*}-b\right)=0, \\
\sqrt{g}(\operatorname{Re} b)^{2}+B \operatorname{Im} b-\sqrt{g}=0
\end{gathered}
$$

при выводе равенств (3.11)-(3.13) считалось, что корень является положительным на продолжении отрезка $\left[-b^{*}, b\right]$ правее $b$. Тогда на оси, перпендикулярной данному отрезку и пересекающей его посередине, выше точки пересечения корень будет чисто мнимым с положительной мнимой частью, а ниже пересечения - чисто мнимым с отрицательной мнимой частью. Пусть $\lambda_{0} \in C$. Учитывая вышесказанное и формулы Сохоцкого-Племеля, получаем

$$
\lim _{\epsilon \rightarrow 0}\left(\Phi\left(\lambda_{0}+i \epsilon\right)-\Phi\left(\lambda_{0}-i \epsilon\right)\right)=2 i\left(A \lambda_{0}+B\right) \sqrt{\left(\lambda_{0}+b^{*}\right)\left(b-\lambda_{0}\right)}=-2 i \pi \bar{v}\left(\lambda_{0}\right),
$$

откуда

$$
\bar{v}(\lambda)=-\frac{1}{\pi}(A \lambda+B) \sqrt{\left(\lambda+b^{*}\right)(b-\lambda)}, \quad \lambda \in C .
$$

Поэтому последнее условие записывается в виде

$$
-\frac{1}{\pi} \int_{-b^{*}}^{b} \frac{A \nu+B}{1-2 i \sqrt{g} \nu} \sqrt{\left(\nu+b^{*}\right)(b-\nu)} d \nu=1 .
$$

Итак, нас интересуют интегралы следующего вида:

$$
\begin{aligned}
& I_{1}=\int_{-b^{*}}^{b} \sqrt{\left(\nu+b^{*}\right)(b-\nu)} d \nu \\
& I_{2}=\int_{-b^{*}}^{b} \frac{d \nu}{1-2 i \sqrt{g} \nu} \sqrt{\left(\nu+b^{*}\right)(b-\nu)} .
\end{aligned}
$$

Они находятся однотипным образом. Фактически интегралы представляют собой интегралы по контуру, совпадающему с верхним берегом разреза и идущему слева направо. Так как на нижнем береге разреза подынтегральная функция принимает противоположное значение, то после замыкания контура по часовой стрелке значение нашего интеграла будет равно половине значения интеграла, взятого по замкнутому контуру. Интеграл по замкнутому контуру в случае $I_{1}$ равен $2 \pi i \times$ (вычет на бесконечности), а в случае $I_{2}$ нужно еще принять во внимание вычет в полюсе. Обозначим $\tau(\nu)=\sqrt{\left(\nu+b^{*}\right)(b-\nu)}$. В результате имеем

$$
\begin{aligned}
& I_{1}=\pi \operatorname{res}(\tau(\nu), \infty), \\
& I_{2}=\pi\left(\operatorname{res}\left(\frac{\tau(\nu)}{1-2 i \sqrt{g} \nu}, \infty\right)+\operatorname{res}\left(\frac{\tau(\nu)}{1-2 i \sqrt{g} \nu}, \nu_{0}\right)\right),
\end{aligned}
$$

где $\nu_{0}=1 /(2 i \sqrt{g})$ - положение полюса. После вычислений получим

$$
I_{1}=\frac{\pi}{2}(\operatorname{Re} b)^{2}
$$




$$
I_{2}=\frac{\pi}{2 \sqrt{g}}\left(\left((\operatorname{Re} b)^{2}+\left(\operatorname{Im} b+\frac{1}{2 \sqrt{g}}\right)^{2}\right)^{1 / 2}-\operatorname{Im} b-\frac{1}{2 \sqrt{g}}\right) .
$$

Заметим, что из определения интеграла $I_{2}(g)$ ясно, что он несингулярен при $g \rightarrow 0$. Формально в полученном явном выражении для $I_{2}$ присутствуют сингулярности, однако легко убедиться, что они сокращаются. Тем самым это является некоторой проверкой наших вычислений. Выпишем явно все полученные нами условия на $B$, $\operatorname{Re} b, \operatorname{Im} b$, сразу исключив $A$ :

$$
\begin{aligned}
B & =-1-2 \sqrt{g} \operatorname{Im} b, \\
0 & =\sqrt{g}(\operatorname{Re} b)^{2}+B \operatorname{Im} b-\sqrt{g}, \\
1 & =\frac{1}{\pi} I_{1}+\frac{2 \sqrt{g} \operatorname{Im} b}{\pi} I_{2} .
\end{aligned}
$$

Как мы видим, уравнения вещественны, вследствие этого число неизвестных совпадает с числом уравнений. Это является подтверждением правильности учета симметрии контура. Выпишем еще раз все уравнения явно, вводя параметр $\varepsilon=1 /(2 \sqrt{g})$ и исключая $B$ :

$$
\begin{aligned}
\frac{1}{2} & =\frac{1}{2}(\operatorname{Re} b)^{2}-(\operatorname{Im} b)^{2}-\varepsilon \operatorname{Im} b, \\
1 & =\frac{1}{2}(\operatorname{Re} b)^{2}-(\operatorname{Im} b)^{2}-\varepsilon \operatorname{Im} b+\operatorname{Im} b \sqrt{(\operatorname{Re} b)^{2}+(\operatorname{Im} b+\varepsilon)^{2}} .
\end{aligned}
$$

Перейдем к пределу $\varepsilon \rightarrow 0$, обозначив $\bar{b}=\lim _{\varepsilon \rightarrow 0} b(\varepsilon)$. Тогда, учитывая уравнение (3.26), получим

$$
\frac{1}{2}=\operatorname{Im} \bar{b} \sqrt{3(\operatorname{Im} \bar{b})^{2}+1}
$$

Решение этого уравнения имеет вид

$$
\operatorname{Im} \bar{b}=\frac{1}{\sqrt{6}}, \quad \operatorname{Re} \bar{b}=\frac{2}{\sqrt{3}} .
$$

Мы видим, что контур $C$ лежит выше вещественной оси, поэтому полюс $\nu_{0}=-i \varepsilon$ никогда не попадает на контур, что и следовало ожидать. Концы контура в пределе $\varepsilon \rightarrow 0$ оказались на конечном расстоянии друг от друга и от начала координат. Это означает, что данный подход правильно отражает асимптотические свойства модели - "схлопывание" контура, которое имело место в (3.3) при $g \rightarrow \infty$, отсутствует. Вернемся теперь к задаче вычисления статистической суммы. Как ясно следует из (3.5), свободная энергия в пределе $N \rightarrow \infty$ имеет вид

$$
\mathcal{E}=\int d \lambda v(\lambda)\left(\lambda^{2}+\ln \left(\frac{1}{2}-i \sqrt{g} \lambda\right)\right)-\int d \lambda d \mu v(\lambda) v(\mu) \ln |\lambda-\mu| .
$$

Из приведенных результатов видно, что существует конечный предел функции $v(\lambda)$, когда $g \rightarrow \infty$. Поэтому основной вклад в $\mathcal{E}$ в указанном пределе дает $\sqrt{g}$, стоящий под знаком первого логарифма. Следовательно,

$$
\mathcal{E} \underset{g \rightarrow \infty}{\rightarrow} \frac{1}{2} \ln g,
$$

что в точности совпадает с аналогичным выражением в векторной модели (2.13). 


\section{4. ОБСУЖДЕНИЕ И ЗАКЛЮЧЕНИЕ}

Итак, в данной работе рассмотрена комплексная $D=0$ модель. В частности, получено выражение для статсуммы с помощью метода, аналогичного методу работы [3]. В старшем порядке по $g$ в пределе сильной связи предположение об аналогии между матричной и векторной моделями оказывается справедливым. Более того, данное предположение будет верно и в следующем порядке, если в векторной модели использовать не заряд $g$, а заряд $\tilde{g}=\alpha \cdot g$ ( $\alpha=$ const $)$, т.е. если совершить конечную перенормировку заряда. Это ясно из общей структуры свободной энергии для обеих моделей:

$$
E=\frac{1}{2} \ln g+\beta_{0}+\frac{\beta_{1}}{\sqrt{g}}+\cdots .
$$

Всегда можно сделать константу $\beta_{0}$ равной любому числу, заменив соответствующим образом $g \rightarrow \alpha \cdot g$. Нетривиальность заключается в том, что сами функциональные структуры в разложении сильной связи матричной и векторной моделей совпадают, коэффициенты же могут и отличаться (несколько первых коэффициентов можно сделать равными соответствующей конечной перенормировкой параметров).

Наше предположение также верно при $D=1$, что нетрудно понять из явного вида асимптотик свободной энергии матричной и векторной моделей: обе они ведут себя как $E \sim \kappa \cdot g^{1 / 3} \quad(\kappa=$ const $)$. Вопрос о том, верно ли наше предположение при $D \geqslant 2$, остается открытым.

Благодарности. Работа частично поддержана РФФИ (грант № 05-01-00541), Программой поддержки ведущих научных школ (грант № НШ-672.2006.1). Д. Быков благодарен профессору М. Мюллеру-Пройсскеру за гостеприимство в Университете им. Гумбольдтов (Берлин, Германия), где была выполнена значительная часть данной работы, Немецкому обществу академических обменов за финансовую поддержку в рамках стипендии им. Леонарда Эйлера, а также фонду “Династия" за финансовую поддержку.

\section{Список литературы}

[1] G. 't Hooft, "Planar diagram field theories", Progress in Gauge Field Theory, Proc. of the NATO Adv. Study Inst. held in Cargèse (September 1-15, 1983), NATO Adv. Science Inst. Ser. B: Phys., 115, eds. A. Jaffe, H. Lehmann, P. K. Mitter et al., Plenum Press, New York, 1984, 271-335.

[2] O. Aharony, S.S. Gubser, J. Maldacena, H. Ooguri, Y. Oz, Phys. Rep., 323:3-4 (2000), 183-386; arXiv:hep-th/9905111.

[3] E. Brézin, C. Itzykson, G. Parisi, J. B. Zuber, Comm. Math. Phys., 59:1 (1978), 35-51.

[4] J. Ambjørn, J. Jurkiewicz, Yu. M. Makeenko, Phys. Lett. B, 251:4 (1990), 517-524.

[5] L. O. Chekhov, J. Phys. A, 39:28 (2006), 8857-8893; arXiv:hep-th/0602013. 